\title{
Evaluation of Trichoderma consortia against Fusarium udum causing wilt of Pigeonpea
}

\author{
Monika Patel \\ Department of Plant Pathology, College of Agriculture, Jawaharlal Nehru Krishi Vishwa \\ Vidyalaya, Jabalpur-482004 (Madhya Pradesh), India \\ Sanjeev kumar* \\ Department of Plant Pathology, College of Agriculture, Office of Dean Faculty of Agriculture, \\ Jawaharlal Nehru Krishi Vishwa Vidyalaya, Jabalpur-482004 (Madhya Pradesh), India \\ ${ }^{*}$ Corresponding address. Email: sanjeevcoa@gmail.com
}

\section{Article Info}

https://doi.org/10.31018/ jans.v13i2.2656

Received: April 4, 2021

Revised: May 13, 2021

Accepted: May 18, 2021

\section{How to Cite}

Patel, M. and Kumar, S. (2021). Evaluation of Trichoderma consortia against Fusarium udum causing wilt of Pigeonpea. Journal of Applied and Natural Science, 13(2), 537 - 543. https://doi.org/10.31018/jans.v13i2.2656

\begin{abstract}
Pigeonpea is one of the important pulse crop of Madhya Pradesh, a State of India. The plant gets infected by the pathogen Fusarium udum causing wilt disease, which is one of the major constraints in the production and productivity of pigeonpea. The present study aimed to carry out in vitro condition to assess the possible use of biocontrol consortia in field conditions. Six Trichoderma consortia viz., $\mathrm{T}_{1}-T$. viride $+T$. harzianum (JC-I), $\mathrm{T}_{2}-T$. viride $+T$. virens $(\mathrm{JC}-2)$, $\mathrm{T}_{3:-} T$. harzianum $+T$. virens $(\mathrm{JC}-$ 3), $\mathrm{T}_{4}-\mathrm{T}$. hamatum $+T$. viride (JC-4), $\mathrm{T}_{5^{-}} \mathrm{T}$. hamatum $+T$. harzianum (JC-5), $\mathrm{T}_{6^{-}} \mathrm{T}$. hamatum $+\quad T$. virens $(\mathrm{JC}-6)$ and $\mathrm{T}_{0-}$ Control were evaluated for their antagonistic activity against $F$. udumunder in vitro conditions. The consortia of $T_{4}-T$. hamatum $+T$. viride (JC-4) was found most effective $(58.82 \%)$ in inhibiting the radial growth of Fusarium udum. The volatile compound from consortium of $\mathrm{T}_{5}-T$. hamatum $+T$. harzianum (JC-5) exhibited maximum growth inhibition $(81.84 \%)$ and sporulation of Fusarium udum followed by $\mathrm{T}_{1}-T$. viride $+T$. harzianum (JC-I) $\left(55.49 \%\right.$ inhibition). The culture filtrate of consortia of $\mathrm{T}_{4^{-}} T$. hamatum $+T$. viride (JC-4) showed $100 \%$ inhibition of test pathogen followed by $T_{5}-T$. hamatum $+T$. harzianum (JC-5) $(82.89 \%)$ at $5 \%$ concentration. It was also observed that with an increase in the concentration of culture filtrates of all the Trichoderma species, the radial mycelial growth of the test pathogen was proportionally decreased. The Trichodema consortium viz., $\mathrm{T}_{4^{-}} T$. hamatum $+T$. viride $(\mathrm{JC}-4)$ may be tried in the field to manage wilt of pigeonpea because they worked synergistically and gave the high impact of their use.
\end{abstract}

Keywords: Consortia, Fusarium udum, Pigeonpea, Trichoderma, Wilt

\section{INTRODUCTION}

Pigeon pea is one of the key pulse crops grown in India. Pigeonpea occupies a unique place on Indian agriculture scene as the country accounts for about $71.5 \%$ of the global production, covering an area of around $5.40 \mathrm{~m}$ ha and production of 4.87 million tonnes. The average productivity of pigeon pea is about $750 \mathrm{~kg} / \mathrm{ha}$, which is much lower than their potential yields. (Singh et al., 2020). Pigeonpea is known to be infected by more than 200 pathogens reported from 23 different countries (Nene et al., 1981). Wilt is predominant in all major pigeon pea growing areas throughout India and causes $30-100 \%$ yield loss (Biswas and Ghosh, 2016). The intensive use of fungicides results in environmental pollution, the resistance of pathogens towards fungicides, hazardous to human and animals. This necessitates the need to adopt sustainable management of disease like using antagonistic fungi against the pathogen. (Harman, 2011; Singh et al., 2011; Kumar et al., 2014).

There are many traditional strategies as well as chemical approaches for managing the wilt disease in pigeon pea. Lesser emphasis on understanding the application of biological approaches for managing Fusarium udum in pigeon pea fields. (Sharma et al., 2012). Over the years, numerous studies have described the application of microbial consortia for plant disease management throughout the world. Studies revealed that plants treated with anagonistic microbial 
consortia showed a significant disease reduction compared to individual isolates. Biocontrol attributes are also more in consortia than using single isolates (Thakkar and Saraf, 2015). Studies on employing indigenous fungal antagonistic consortia are very limited against the wilt of pigeonpea in Madhya Pradesh. Concerning this, the present study was focused on the approach to evaluating Trichoderma consortium against Fusarium udum that could help in effective management of wilt of pigeonpea.

\section{MATERIALS AND METHODS}

\section{Isolation and identification of test pathogen}

Pigeonpea plants showing typical symptoms of Fusarium wilt were collected from the experimental field of Jawaharlal Nehru Krishi Vishwa VidyalayaJabalpur for isolation and identification. The infected plant parts were cut into small pieces and surface sterilized with 0.1 per cent Sodium hypochloride solution and washed thoroughly 3 to 4 times with sterilized water to remove the traces of sodium hypochlorite. The pieces were transferred in petri dishes containing potato dextrose agar medium and incubated at $27 \pm 1^{\circ} \mathrm{C}$ for 7 days. The pure culture was isolated from inoculated petriplates separately in aseptic condition.( Chaudhary et al., 2017).

The following six Trichoderma consortium were evaluated to test the antagonism against Fusarium udum in the Department of Plant Pathology Jawaharlal Nehru Krishi Vishwavidyalaya, Jabalpur (M.P.) during 202021.

$\mathrm{T}_{1}$-Trichoderma viride + Trichoderma harzianum (JC-I)

$\mathrm{T}_{2}$-Trichoderma viride + Trichoderma virens(JC-2)

$\mathrm{T}_{3:}$-Trichoderma harzianum + Trichoderma virens(JC-3)

$\mathrm{T}_{4}$-Trichoderma hamatum +Trichoderma viride (JC-4)

$\mathrm{T}_{5}$-Trichoderma hamatum + Trichoderma harzianum (JC-5)

$\mathrm{T}_{6}$-Trichoderma hamatum + Trichoderma virens $(\mathrm{JC}-6)$

$\mathrm{T}_{0 .}$. Control

The Trichoderma used in the consortium were isolated and identified in the laboratory as per the method given by Gams and Bisset (1998) and will be submitted to Indian type culture collection, Division of Plant Pathology, Indian Institute of Agriculture Research (IARI), New Delhi for accession numbers.

\section{Effect of Trichoderma consortia on the radial} growth of $F$. udum in dual culture

A dual culture technique developed by Morton and Straube (1955) and Patole et al (2017) was adopted to study the effect of Trichoderma consortia against $F$. udum,. Twenty $\mathrm{ml}$ sterilized melted PDA media was poured into sterilized petriplates @ 20 ml/plate aseptically, allowed to solidify. For the Trichoderma consortium, $8 \mathrm{~mm}$ disc of Trichoderma was placed at equidis- tance on potato dextrose agar media in a petriplate. Immediately after inoculation, the plates were sealed with plastic film and incubated at $27 \pm 1^{\circ} \mathrm{C}$ for 1 week period. Observations were recorded after $3,5,7$ days of inoculation on the growth of individual Trichoderma in the presence of its co-inoculant. Pairs of consortia were considered compatible if they grow without any inhibition zone in the culture plate. Then, $8 \mathrm{~mm}$ disc of test pathogen and the consortia cut with the help of sterilized cork borer were placed on PDA approximately 4 $\mathrm{cm}$ apart from each other and incubated in BOD incubator at $27 \pm 1^{\circ} \mathrm{C}$ for 144 hours. Three replications were maintained for each treatment. Observations on colony diameter of individual antagonist and the pathogen were recorded after 144 hours of incubation. Inhibition of radial growth of $F$. udum over control was calculated by the formula given by Vincent (1947).

\section{Effect of volatile and non volatile compounds from Trichoderma consortia on the radial growth of Fusarium udum}

The effect of volatile compounds from Trichoderma consortia viz., $\mathrm{T}_{1}-T$. viride $+T$. harzianum $(\mathrm{JC}-\mathrm{I}), \mathrm{T}_{2}-T$. viride $+T$. virens $(\mathrm{JC}-2), \mathrm{T}_{3}:-T$. harzianum $+T$. virens (JC-3), $\mathrm{T}_{4^{-}} T$. hamatum $+T$. viride (JC-4), $\mathrm{T}_{5}-T$. hamatum $+T$. harzianum (JC-5) and $T_{6}-T$. hamatum + $T$. virens (JC-6) on radial growth of test pathogen was performed as per the method given by Dennis and Webster (1971a and1971b). Two bottom portion of petridplates containing potato dextrose agar were inoculated with $8 \mathrm{~mm}$ disc of test pathogen and Trichoderma consortia, respectively and both inoculated bottom plates were placed facing each other and sealed with cellophane adhesive tape. The petriplate containing PDA without antagonist served as control. The observations on the radial growth of the test pathogen were recorded after $144 \mathrm{~h}$ of incubation at $27 \pm 1^{\circ} \mathrm{C}$.

To study the effect of non volatile compounds, the Trichoderma consortia were grown on potato dextrose broth at $27 \pm 1^{\circ} \mathrm{C}$ with intermittent shaking at $150 \mathrm{rpm}$. Themetabolites were collected after 15 days and filtered. The sterilized filtrate was amended in potato dextrose agar to make 2 and $5 \%$ concentration in petriplates. The solidified agar plates were inoculated at the centre with $5 \mathrm{~mm}$ mycelial disc of pathogen and incubated at $27 \pm 1^{\circ} \mathrm{C}$ for 96 hours. The plates without filtrate served as control. Observations on radial growth of individual Trichoderma consortia and the test pathogen were recorded after 96 hours of incubation. Inhibition of radial growth of test pathogen over control was calculated by formula given by Vincent (1947).

$$
\begin{aligned}
& \text { Percent growth } \\
& \text { inhibition }(\mathrm{I})
\end{aligned}=\frac{\mathrm{C}-\mathrm{T}}{\mathrm{C}} \times 100
$$


Patel, M. and Kumar, S. / J. Appl. \& Nat. Sci. 13(2), 537 - 543 (2021)

Where,

$\mathrm{C}=$ Radial growth in check plate $(\mathrm{mm})$

$\mathrm{T}=$ Radial growth in the treated plate $(\mathrm{mm})$

\section{RESULTS AND DISCUSSION}

\section{Compatibility among Trichoderma}

All the six Trichoderma consortium (JC-1, JC-2, JC-3, JC-4, JC-5 \& JC-6) were found compatible with each other as no isolates inhibited the growth of one another. They were growing simultaneously on the PDA without inhibiting the growth of other or formation of inhibition zone in their combinations. All the six Trichoderma consortium were found compatible with each other as no isolates inhibited the growth of one another. They were growing simultaneously on the PDA without inhibiting the growth of other or formation of inhibition zone in their combinations.

\section{Evaluation of antagonistic efficacy of consortium} of Trichoderma against $F$. udum

The consortium of $\mathrm{T}_{4^{-}} T$. hamatum $+T$. viride (JC-4) was found most effective(58.82 \%) in inhibiting the radial growth of Fusarium udum (Table-1, and Plate1)The percent growth inhibiton recorded in $T_{2^{-}} T$. viride $+T$. virens $(\mathrm{JC}-2), \mathrm{T}_{3}-T$. harzianum $+T$. virens (JC-3) and $T_{5}-T$. hamatum $+T$. harzianum (JC-5) were, respectively, $57.20,56.79,56.38 \%$ and were statistically at par with each other. The percent inhibition recorded in $\mathrm{T}_{1}-T$. viride $+T$. harzianum (JC-I) and $T_{6}-T$. hamatum $+T$. virens (JC-6) were 44.21 and $19.83 \%$,respectively.

Evaluation of antagonistic efficacy of volatile and non volatile compounds from consortium of Trichoderma against Fusarium udum

The volatile compound from consortium of $T_{5}-T$. hamatum $+T$. harzianum (JC-5) exhibited maximum growth inhibition (81.84\%) and sporulation of Fusarium udum followed by $\mathrm{T}_{1}-T$. viride $+T$. harzianum $(\mathrm{JC}-\mathrm{I})$ $(55.49 \%)$ and $\mathrm{T}_{4}-\mathrm{T}$. hamatum $+T$. viride (JC-4) $(43.23$ $\%)$ (Table-2, and Plate-2). $T_{6}-T$. hamatum $+T$. virens (JC-6) and $\mathrm{T}_{2}-T$. viride $+T$. virens (JC-2) inhibited $35.13 \%$ and $34.74 \%$ growth of $F$. udum. Minimum growth inhibition $29.54 \%$ recorded in $T_{3:-} T$. harzianum $+T$. virens (JC-3) after 144 hours of incubation.

The culture filtrate of consortia of $\mathrm{T}_{4}-T$. hamatum $+T$. viride (JC-4) showed cent per cent inhibition of test pathogen followed by $T_{5}-T$. hamatum $+T$. harzianum (JC-5) (82.89\%) at $5 \%$ concentration (Table-3, and Plate- 3 ) . $\mathrm{T}_{2}-T$. viride $+T$. virens (JC2) and $T_{6}-T$. hamatum $+T$. virens (JC-6) exhibited $78.52 \%$ and $77.63 \%$ mycelial inhibition of test pathogen and statistically at par with each other.The consortia of $\mathrm{T}_{3:}-T$. harzianum $+T$. virens (JC-3), and $\mathrm{T}_{1}-T$. viride $+T$. harzianum (JC-I) exhibited respectively 67.10 and $61.84 \%$ growth inhibition of $F$. udum. The test pathogen exhibited $38.00 \mathrm{~mm}$ growth after 96 hour of incubation.It was also observed that with an increase in concentration of culture filtrates of all the Trichoderma consortia, the radial mycelial growth of test pathogen was proportionally decreased.

Over the years, numerous studies have described the application of microbial consortia for plant disease management throughout the world. Studies revealed that plants treated with anagonistic microbial consortia showed a significant disease reduction when compared to using individual isolates (Nikam et al., 2007, Sharma et al., 2012,Patoleet al., 2017). The principal biocontrol mechanisms involved include mycoparasitism, antibiosis, competition, and induced resistance ( Kumar et al. 2009, Kumar. 2013, Chaudhary et al., 2017, Kushwaha et al., 2018). Application of bioagents in a consortium may improve the efficacy, reliability and consistency of the bioagents even under diverse soil and environmental conditions (Sharma et al. 2012; Amirthalingam et al.

Table 1. Evaluation of antagonistic efficacy of consortium of Trichoderma against $F$. udum.

\begin{tabular}{llll}
\hline T. No . and Treatment Name & $\begin{array}{l}\text { Radial growth of } \\
\text { test Pathogen }(\mathbf{m m})\end{array}$ & $\begin{array}{l}\text { Percent growth } \\
\text { inhibition }\end{array}$ & Sporulation \\
\hline $\mathrm{T}_{1}$ T. viride $+T$. harzianum & 45.83 & 44.21 & +++ \\
$\mathrm{T}_{2}$ T. viride $+T$. virens & 35.16 & 57.20 & +++ \\
$\mathrm{T}_{3}$ T. harzianum $+T$. virens & 35.50 & 56.79 & +++ \\
$\mathrm{T}_{4}$ T. hamatum $+T$. viride & 33.83 & 58.82 & + \\
$T_{5}$ T. hamatum + T. harzianum & 35.83 & 56.38 & +++ \\
$T_{6}$ T. hamatum + T. virens & 65.86 & 19.83 & +++ \\
$\mathrm{T}_{0:}$ Control & 82.16 & & +++ \\
$\mathrm{SE}(\mathrm{m})$ & 0.78 & & \\
$\mathrm{CD}$ & 2.10 & & \\
\hline
\end{tabular}

${ }^{*}$ Average of three replications 

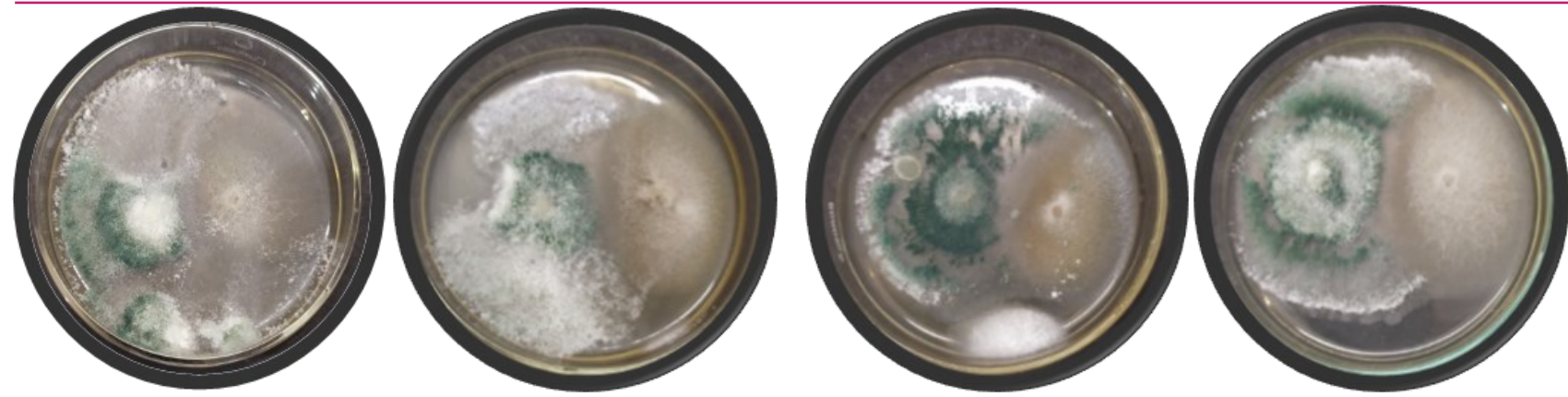

T. viride + T. harzianum T. viride + T. virens

T. harzianum + T. virens

T. hamatum + T. viride

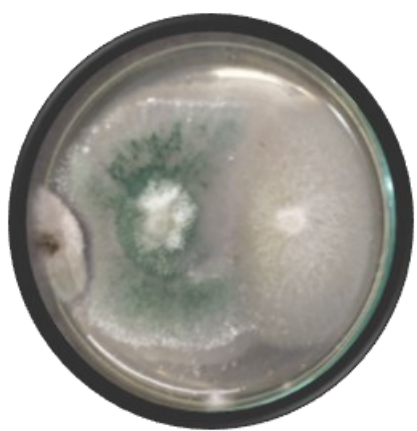

$T$. hamatum $+T$. harzianum

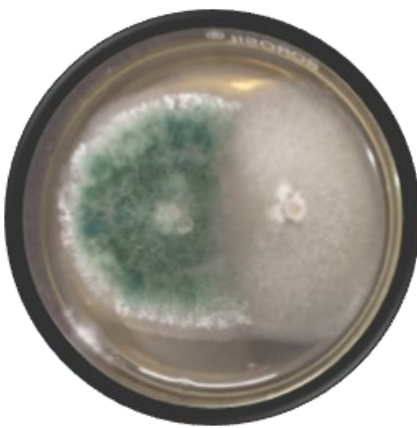

T. hamatum + T. virens

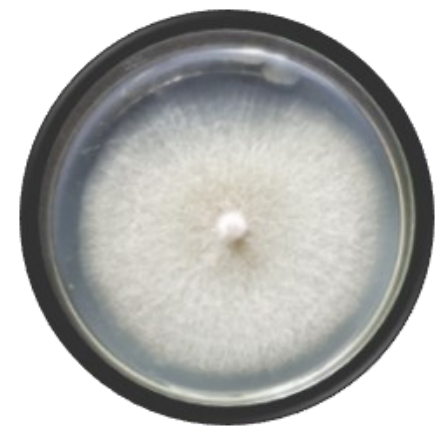

Control

Plate 1: Evaluation of antagonistic efficacy of consortium of Trichoderma against Fusarium udum.

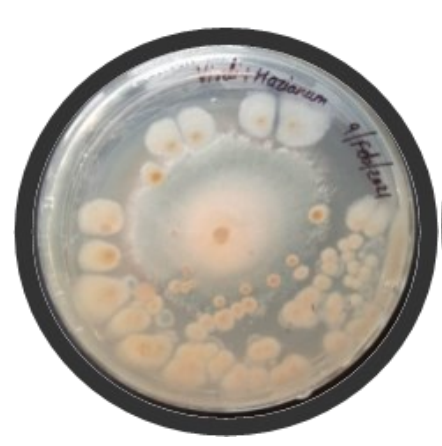

T. viride + T. harzianum

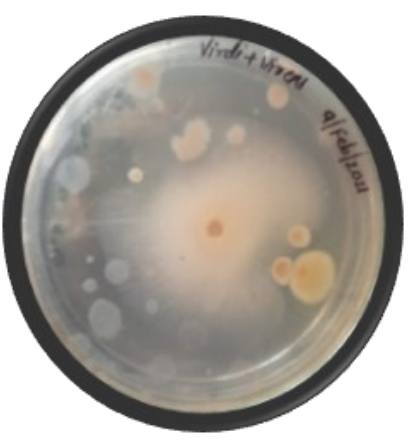

$T$. viride $+T$. virens

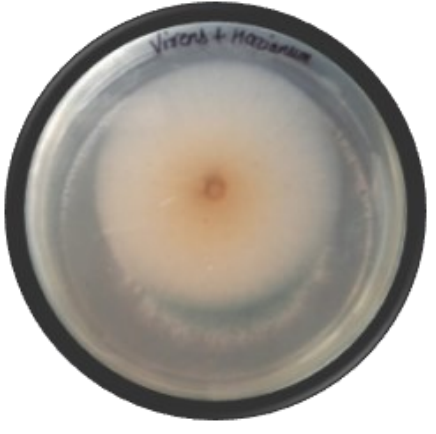

T. harzianum + T. virens

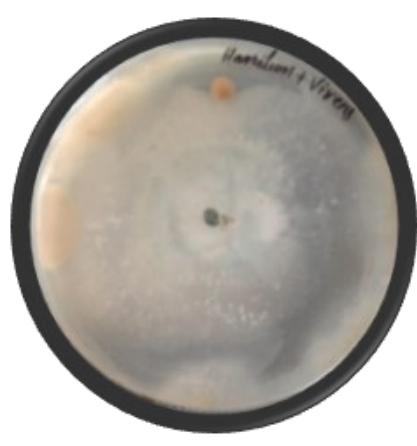

T. hamatum + T. viride

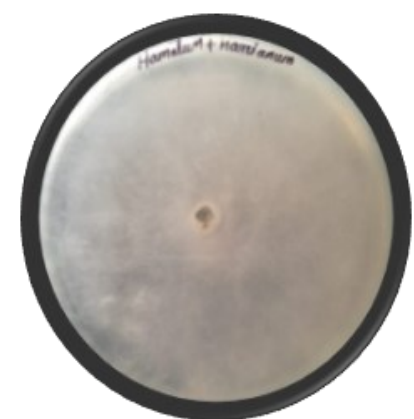

T. hamatum + T. harzianum

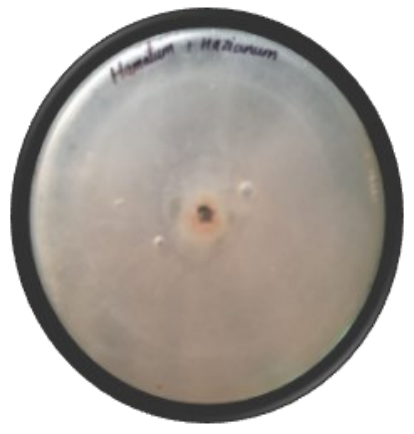

T. hamatum + T. virens

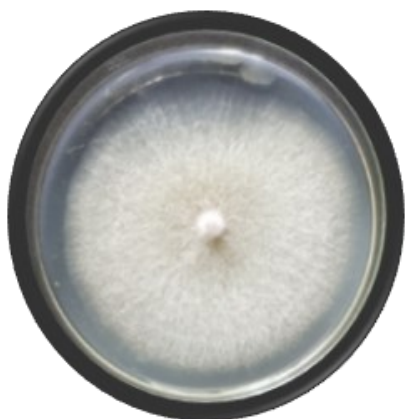

Control

Plate 2. Showing antagonistic efficacy of volatile compound from consortium of Trichoderma against Fusarium udum.

2020). A consortium of $T$. asperellum GDFS1009 and Bacillus amyloliquefaciens ACCC1111060 was found to be more efficient against infection by Botrytis cinerea, causing grey mold disease than the individual strains (Wu et al., 2019). Likewise, when Trichoderma virens GI006 was combined with Bacillus velezensis Bs006, efficiency against Fusarium wilt of cape gooseberry was enhanced (Izquierdo-García et al., 2020). Thakkar and Saraf (2015) reported that different biocontrol mechanisms offered by each bioagent in the consortium may help in enhancing pathogen inhibition and may also strengthen the capacity of the partners in an additive or synergistic manner. However, Kumar and 


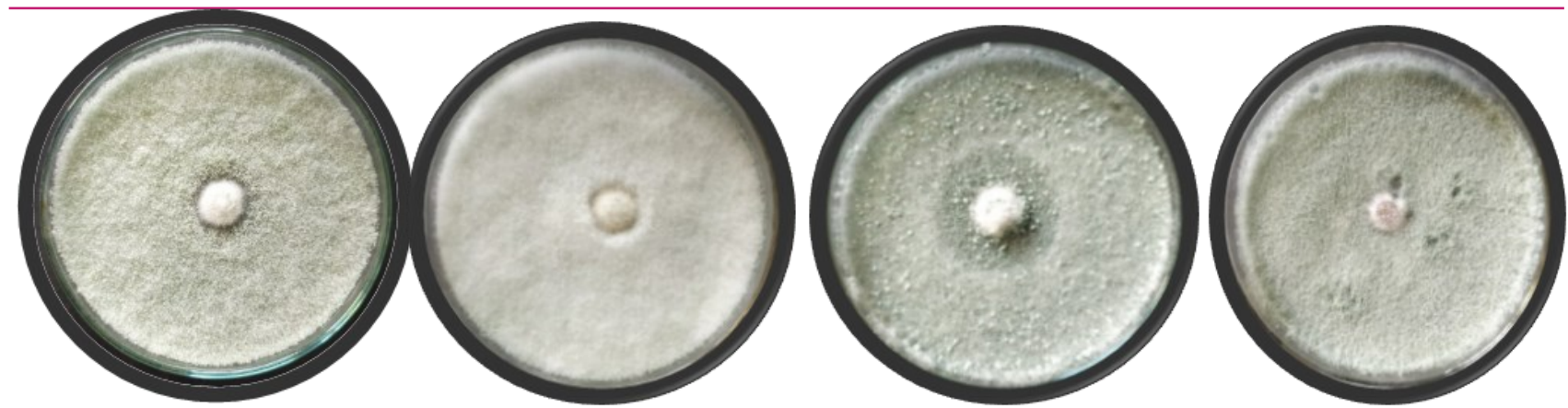

T. viride + T. harzianum T. viride + T. virens

T. harzianum + T. virens T. hamatum + T. viride

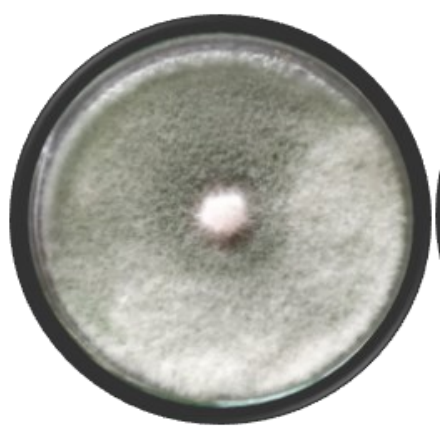

$T$. hamatum + T. harzianum

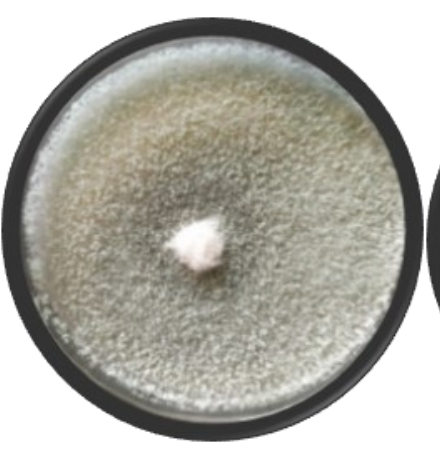

T. hamatum + T. virens

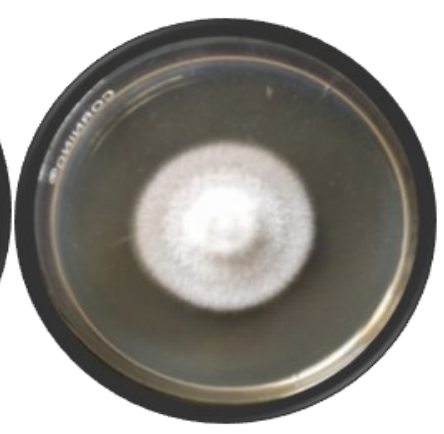

Control

Plate 3 . Showing efficacy of non volatile compound from consortium of Trichoderma against Fusarium udum .

Table 2: Evaluation of antagonistic efficacy of volatile compound from consortium of Trichoderma spp. against $F$. udum under in vitro condition.

\begin{tabular}{llll}
\hline T. No. and Treatment Name & $\begin{array}{l}\text { Radial growth of test } \\
\text { Pathogen }(\mathbf{m m})^{\#}\end{array}$ & $\begin{array}{l}\text { Percent growth } \\
\text { inhibition }\end{array}$ & Sporulation \\
\hline $\mathrm{T}_{1}$ T. viride + T. harzianum & 37.16 & 55.49 & ++ \\
$\mathrm{T}_{2}$ T. viride + T. virens & 54.50 & 34.74 & +++ \\
$\mathrm{T}_{3}$ T. harzianum+ T. virens & 58.83 & 29.54 & +++ \\
$\mathrm{T}_{4}$ T. hamatum + T. viride & 48.16 & 43.23 & +++ \\
$\mathrm{T}_{5}$ T. hamatum + T. harzianum & 15.16 & 81.84 & - \\
$\mathrm{T}_{6}$ T. hamatum + T. virens & 54.16 & 35.13 & +++ \\
$\mathrm{T}_{7}$ Control & 83.50 & & + \\
$\mathrm{SE}(\mathrm{m})$ & 0.930 & & \\
$\mathrm{CD}$ & 2.84 & & +++ \\
\hline
\end{tabular}

${ }^{*}$ Average of three replications

Jagadeesh (2016) reported that certain microbial consortia were unable to show at least comparable effects on plants with respect to their individual applications, which may be attributed to the incompatibility of the microbes in the mixture with each other and do not have any additive or synergistic effects on disease suppression.

\section{Conclusion}

The application of Trichoderma consortia viz., $T$. hamatum $+T$. viride can be more useful than the indi- vidual for management of wilt of pigeonpea because of two compatible isolates of different species work synergistically and gave the high impact of their use. Reduction in disease by using Trichoderma consortia may reduce the chemical pesticide loads on the pigeonpea crop, which will be in favour of farmers and consumers as well.

\section{Conflict of interest}

The authors declare that they have no conflict of interest. 
Table 3. Effect of non-volatile compounds from consortium of Trichoderma on growth and sporulation of $F$. udum.

\begin{tabular}{|c|c|c|c|c|}
\hline \multirow[t]{2}{*}{ T. No. and Treatment Name } & \multicolumn{2}{|c|}{$\begin{array}{c}\text { Radial growth of test } \\
\text { Pathogen after } 96 \mathrm{hrs}(\mathrm{mm})^{*}\end{array}$} & \multirow{2}{*}{$\begin{array}{l}\text { Percent growth } \\
\text { inhibition }\end{array}$} & \multirow[t]{2}{*}{ Sporulation } \\
\hline & $2 \%$ & $5 \%$ & & \\
\hline $\mathrm{T}_{1} T$. viride $+T$. harzianum & 8.66 & 14.50 & 61.84 & +++ \\
\hline $\mathrm{T}_{2} T$. viride $+T$. virens & 11.67 & 8.16 & 78.52 & ++ \\
\hline $\mathrm{T}_{3} T$. harzianum+ T.virens & 15.83 & 12.50 & 67.10 & +++ \\
\hline $\mathrm{T}_{4} T$. hamatum $+T$. viride & 0.00 & 0.00 & 100.00 & - \\
\hline $\mathrm{T}_{5} T$. hamatum $+T$. harzianum & 8.83 & 6.50 & 82.89 & + \\
\hline $\mathrm{T}_{6}$ T. hamatum $+\quad$ T. virens & 12.50 & 8.50 & 77.63 & + \\
\hline $\mathrm{T}_{7}$ Control & 36.83 & 38.00 & & ++++ \\
\hline $\mathrm{SE}(\mathrm{m})$ & 0.40 & 0.454 & & \\
\hline CD & 1. 23 & 1.39 & & \\
\hline
\end{tabular}

*Average of three replications

\section{REFERENCES}

1. Amirthalingam, V., Tewari, A.K., Sharma, M., Sharma R., \& Kumar, J. (2020). Evaluation of bioagents for their compatibility in the development of consortium for enhanced efficiency. J. Bio. Cont., 34(2), 164-167, 2020, DOI: $10.18311 / \mathrm{jbc} / 2020 / 23179$

2. Biswas, K. \& Ghosh, P.(2016), Recent Advancements and Biological Management of Fusarium udum: A causative agent of Pigeon pea wilt. J. App. \& Natu. Sci., 5(3), $57-72(20$

3. Chaudhary, B., Kumar,S \& Kushwaha, S. K. (2017). Bioefficacy of Trichoderma species against Pigeonpea wilt pathogen. J. App. \& Nat. Sci., 9(4), 2327-2331. https:// doi.org/10.31018/jans.v9i4.1531

4. Dennis, C. \& Webster, J. (1971a). Antagonistic properties of species groups of Trichoderma II. Production of volatile antibiotics. Trans. Brit. Mycol. Soci., 57, 41-48.

5. Dennis, C. and Webster, J. (1971b). Antagonistic properties of species groups of Trichodermal. Production of non volatile antibiotics. Trans. Brit. Mycol. Soci., 57, 25-39.

6. Izquierdo-García, L. F., González-Almario, A., Cotes, A. M.,\& Moreno-Velandia, C. A. (2020). Trichoderma virens GI006 and Bacillus velezensis Bs006: a compatible interaction controlling Fusarium wilt of cape gooseberry. Sci. Rep., 10,6857. doi: 10.1038/s41598020-63689-y

7. Gams, W \& Bisset, J. (1998). Morphology and identification of Trichoderma In: Harman G. E. and Kubicek C. P. (eds). Trichoderma \& Gliocladium. Vol 1 Taylor and Francis, London.

8. Harman, G.E. (2011) Multifunctional fungal plant symbionts: new tools to enhance plant growth and productivity. New Phyto. Comm, For. (3), 647-649

9. Kumar, K.H.\& Jagadeesh, K.S. (2016). Microbial consortia-mediated plant defense against phytopathogens and growth benefits. Sou. Ind. J. Biol. Sci., 2(4), 395-403

10. Kumar, S. (2013). Trichoderma; A biological weapon for managing plant diseases and promoting sustainability. Int. J. Agric. Sci. Vet. Med., 1, 1-16.

11. Kumar, S., Thakur, M \& Rani, A. (2014). Trichoderma: Mass production, formulation, quality control, delivery and its scope in commercialization in India for the man- agement of plant diseases. Afr. J. Agric. Res., 9, 38383852.

12. Kumar, S., Upadhyay, J.P. and Rani, A. (2009). Evaluation of Trichoderma species against Fusarium udum Butler causing wilt of Pigeon pea. J. Biol. Con., 23: 329-332. Kushwaha, S. K., Kumar, S \& Chaudhary, B. (2018). Efficacy of Trichoderma against Sclerotium rolfsii causing collar rot disease of lentil under in vitro conditions. $J$. App. \& Nat. Sci., 10(1), 307-312. https://doi.org/10.31 018/jans.v10i1.1622

13. Morton,D.T\& Straube, N.H. (1955). Antagonistic and stimulatory effects of microorganisms Sclerotium rolfsii. Phytopatho., 45: 419-420.

14. Nene, Y.L., Kannaiyan, J. and Reddy, M.V. (1981). Pigeonpea diseases: resistance screening techniques. Infor. Bulletin No.9. ICRISAT, India.

15. Nikam, P.S., Jagtap, G.P \& Sontakke, P.L.(2007.) Management of chickpea wilt caused by Fusarium oxysporium f. sp. ciceri. Afr. J. Agri. Res., 2(12), pp.692-697.

16. Patole, S.P., Dhore, S.B., Pradhan, R.S. \& Shankara, K. (2017). In vitro evaluation of Trichoderma viride and Trichoderma harzianum against Fusarium wilt of Chickpea, Int. J. Pure App. Biosci., 5(5): 460-464 doi: http:// dx.doi.org/10.18782/2320-7051.5508

17. Singh , A .I., Formm, G.K., Jha, P. Venkatesh, H., Tewari, R., Padaria, U., Egger .(2020). Understanding pigeonpea (Cajanus cajan) production conditions, stakeholders preferences for varietal traits and their implications for breeding programmes in India. bioRxiv 2020.0 6.08.139832; doi: https://doi.org/10.1101/2020.06.08.13 983

18. Singh, B.N., Singh, A., Singh, S.P. \& Singh, H.B. (2011) Trichoderma harzianum-mediated reprogramming of oxidative stress response in root apoplast of sunflower enhances defence against Rhizoctonia solani. Euro. J. PI. Patho. 131(1): 121-134.

19. Thakkar, A. \& Saraf, M. (2015). Development of microbial consortia as a biocontrol agent for effective managementof fungal diseases in Glycine max L. Arch Phytopathol. Plant Prot. 48: 459-474.

20. Sharma, M., Rathore, A., Mangala, U. N., Ghosh, R., Sharma, S. \& Upadhyay H. D. (2012). New sources of resistance to Fusarium wilt and sterility mosaic disease in 
Patel, M. and Kumar, S. / J. Appl. \& Nat. Sci. 13(2), 537 - 543 (2021)

a mini-core collection of pigeonpea germplasm. Eur. J. Plant Pathol. 133, 707-714. 10.1007/s10658-012-9949-9

21. Vincent, J.M. (1947). Distortion of fungal hyphae in presence of certain inhibitors. Nature, 154:8
22. Wu, Y., Zhou, J., Li, C., \& Ma, Y. (2019). Antifungal and

23. plant growth promotion activity of volatile organic compounds produced by Bacillus amyloliquefaciens. Microbiologyopen, 8(8),e0081310.1002/mbo3.813 